\title{
COMBINED COMPONENT SWAPPING MODULARITY FOR A VCT ENGINE CONTROLLER
}

\author{
Melih Çakmakcı* \\ Department of Mechanical Engineering \\ Bilkent University \\ Ankara, 06800, TURKEY \\ Email: cakmakci@umich.edu
}

\author{
A. Galip Ulsoy \\ Department of Mechanical Engineering \\ University of Michigan \\ Ann Arbor, Michigan, 48109-2125 \\ Email: ulsoy@umich.edu
}

\begin{abstract}
The use of bi-directional communication provides additional design freedom which can be used to maximize the swapping modularity of networked smart components. In this paper, application of a design method for combined swapping modularity of two or more system components is discussed. Development of measures for combined swapping modularity is important to be able to analyze more realistic engineering cases. The combined modularity problem is a more difficult problem compared to the individual component swapping modularity problem. First, two approaches (simultaneous and sequential) for combining component swapping modularity of two or more components are presented. Then these combined modularity approaches are used to design controllers which maximize the component-swapping modularity of the Variable Camshaft Timing (VCT) component (i.e. actuator and sensor) and the Exhaust Gas Oxygen (EGO) sensor for an internal combustion engine.
\end{abstract}

\section{INTRODUCTION}

Availability of on-board electronics has increased the use of "smart" components in automatic control systems. Smart components with networking capabilities open up the possibility of using bi-directional communications among components as shown in Fig. 1. The use of bi-directional communications provide additional design freedom which can be used to design control systems with better overall characteristics.

In [1], a method to design control systems where component swapping modularity is maximized using bi-directional communications was presented. Component-swapping modular-

\footnotetext{
*Address all correspondence to this author.
}

ity occurs when two or more alternative basic components can be paired with the same modular components creating different product variants belonging to the same product family [2]. Control systems with modularly swappable components can be defined as the systems in which the initial and final configurations due to a component change operate at their corresponding optimal performance.

As the first step of the method an overall controller, $C$, is designed using conventional design methods, then this controller is distributed to smart component controllers. The resulting distributed controller structure and optimal parameters maximize the component-swapping modularity while providing the same performance as the desired controller, $C$. The proposed method is then successfully applied to maximize the actuator swapping modularity of a simple continuous SISO problem [1].

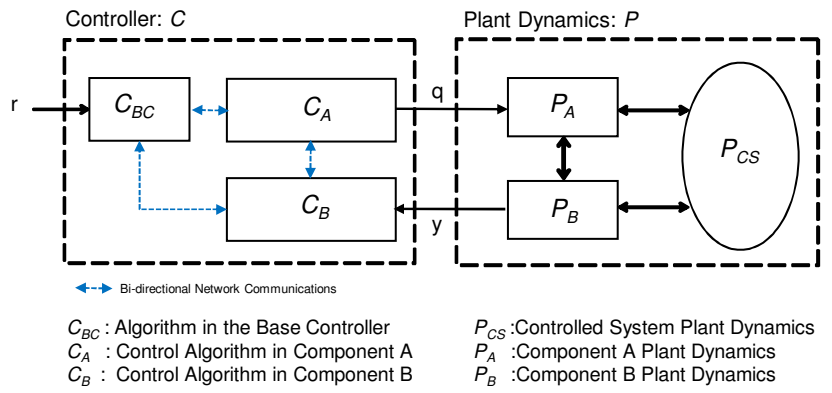

Figure 1. Bi-directional Communications and Overall Control and Plant Composition.

In [3], the design method presented in [1] is applied to a 
more complex problem of design and distribution of discrete MIMO control of a Variable Camshaft Timing (VCT) Engine. The engine plant model is developed based on the work presented in [4] and [5], and an overall discrete-time MIMO controller was designed based on [6]. After formulating the distribution problem and a pre-optimization analysis to simplify the numerical solution of the problem, two distinct optimal distribution solutions optimizing the component swapping modularity of the VCT component and Exhaust Gas Oxygen (EGO) sensor is given respectively.

In [1], four possible use cases for systems designed to have component-swapping modularity are outlined:

\section{Sustainable maintenance and/or upgrade of a} particular end product: Increasing component swapping modularity shortens the engineering time and effort (i.e. cost) in the iterative phases of conceptual design, implementation and testing/validation after each maintenance and upgrade of the system. Many manufacturing facilities house custom made machining systems that only produce a certain type of a product with high accuracy and precision. Building these systems with component swapping modularity in mind would pay off over time through their life cycle since, maintenance and upgrade of a swappable component (such as a "smart" electric motor for an axis manipulator) will not only be easier to perform, but also will result in the best performance possible from the overall system depending on the new component specifications.

2. Deploying controlled system platform based algorithms: Use of platform engineering has been on the rise in recent years for companies which produce a variety of products. Product platforms require defining a common infrastructure with different component variants within a company's product line. Quality of control engineering can be increased drastically by focusing on designing control algorithms for product platforms (more engineering time, focus and experience) which will increase the overall performance of the end-product. Today, many automotive companies develop their vehicles based on vehicle platforms (such as small, midsize, truck, etc.) but offer variants which appeal to different customer bases. For example, many companies offer economy and luxury vehicles based on their small car platform. These two options would present two different cost structures due to the difference in the planned sale price. In parallel, this price difference also forces companies to use better performing alternatives of some components in the luxury option due to better performance expectancy from the vehicle sold in the luxury car segment. Designing these components to be swappable will decrease the engineering and development costs drastically with no impact of the best expected performance from both the economy and luxury version of the same platform design.

3. Deploying control algorithms for different builds

of the same product: For companies which use many different suppliers and operate in many different locations, different builds of the same product are needed because of subsystem variance and difference in system specifications due to regulations, local requirements, etc. For these type of global products, having component swapping modularity in control systems increases the overall efficiency of engineering by obtaining location specific optimal algorithms without redesigning and re-calibrating the whole system. Ever increasing competition in the automotive industry forces many companies to launch so called "global" vehicles, i.e., very appealing vehicle designs built in different parts of the world using the local automotive supply chain and conforming to local regulations. Global vehicles designed with swappable components minimize the re-design efforts to launch a vehicle locally and the need to maintain multiple engineering teams at different parts of the world to solve the same design problem with different constraints.

4. Reducing costs by developing highly customizable but less variant components: Supplier companies which supply sub-systems to more than one company can develop control systems with component swapping modularity to focus on systems which optimally work on many customer end-products. In the automotive industry, many components such as EGO Sensors are primarily provided by suppliers. Getting specifications for the next "smart" EGO sensor right (i.e. right amount of computing power with the right networking bus) would improve the competitiveness of the supplier considerably since these components are produced in bulk and sold to multiple auto manufacturers (OEMs).

The cases outlined above imply either design or deployment of systems belonging to the same or similar product families (Cases 2,3) or upgrade and/or maintenance of a single system (Case 1). In the studies discussed earlier (i.e [1] and [3]), component swapping modularity of only one component at a time is considered. Single component focused component swapping modularity studies can be useful for a scenario similar to Case 4 . However, other realistic engineering scenarios require consideration of multiple components while obtaining an optimal distribution and correspond to Cases 1-3 above. In the next sections of this paper, a simultaneous approach and a sequential approach, which are suitable for Cases 2-3 and Case 1 respectively for calculating the combined system modularity, will be presented.

In this paper, generalization of the design method presented in $[1,3]$ to the combined swapping modularity of system components will be presented. In the next section, two approaches (i.e. simultaneous and sequential) for combining component swapping modularity of two or more components are presented. Next, these combined modularity approaches are used to design controllers which maximize the component-swapping modularity of the VCT Component (i.e. actuator and sensor) and the EGO Sensor for the example given in [3]. The designed distributed controllers utilize the bi-directional communications introduced earlier and exhibit improved combined swapping modularity than 
the traditional centralized version of the controller. This paper concludes with a summary of the results, conclusions and future work.

\section{COMBINED COMPONENT-SWAPPING MODULARITY OF A SYSTEM}

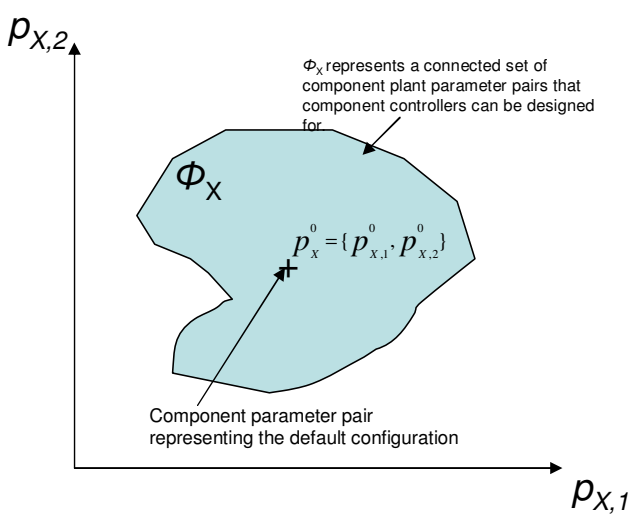

Figure 2. Illustration of Set $\Phi_{X}$ for a Two Parameter Component $X$, $\mathbf{p}_{X}=\left\{p_{X, 1}, p_{X, 2}\right\}$.

The mathematical formulation used to quantify the component swapping modularity for a single component was given in [1]. In summary, as illustrated in Fig. 2 for a component $X$ whose dynamic equations can be represented in terms of two parameters (i.e. $\mathbf{p}_{X}=\left\{p_{X, 1}, p_{X, 2}\right\}$ ), $\Phi_{X}$ is a connected set of component plant parameters including the default parameter value, $\mathbf{p}_{X}^{0}$, that can be achieved by changing only the control parameters for component $\mathrm{X}$ within their limits given a viable distribution solution for the controller.

We then define the function $M_{X}$, the swapping modularity for component $\mathrm{X}$ as

$$
M_{X}\left(\Phi_{X}, \mathbf{p}_{X}\right)=\int_{\Phi_{X}} d \mathbf{p}_{X}
$$

\section{Simultaneous Approach}

One way of combining component swapping modularity is to add the component swapping modularity of components (i.e. $M_{X}$ given in (1)) with appropriate weighing factors (i.e. $\rho_{X}$ ) as shown in (2) for a two component system with components A and $\mathrm{B}$ :

$$
\begin{aligned}
& M_{s y s}\left(\Phi_{A}, \Phi_{B}, \mathbf{p}_{A}, \mathbf{p}_{B}\right)= \\
& \rho_{A} M_{A}\left(\Phi_{A}, \mathbf{p}_{A}\right)+\rho_{B} M_{B}\left(\Phi_{B}, \mathbf{p}_{B}\right)
\end{aligned}
$$

This way of simultaneously calculating the component swapping modularity of components for a particular distribution configura- tion is useful for cases when modularity of a baseline controller design (with the distributed structure) is evaluated. For example, for the cases described in 2 and 3 in the previous section, the baseline design can be used for different product platforms (i.e for example different controlled systems) using the same smart components in the product line (Case 2) or for different instrument configurations (i.e. for example smart components with different performance) due to local availability or requirements in a global deployment situation (Case 3).

For the case of updating two or more components at the same time, it is possible to consider these components as a single component for modularity analysis purposes and use the method described in [1] and [3] to calculate swapping modularity.

\section{Sequential Approach}

When combined modularity of a specific system design rather than a baseline design is considered, adopting sequential a approach while calculating the combined modularity of the system could be more useful. For example, combined swapping modularity calculated by using the previously discussed simultaneous approach offers little information about the modularity of the system for sequential change of components over a time period since once the first component is changed, the original the configuration of the system is lost. This corresponds to Case 1 in the previous section when components of a particular end product are renewed due to failure and/or scheduled maintenance or upgrades.

In these problems a measure for combined modularity of components formulated based on their sequential update can be calculated. For example, if the order of the update is given as update component $A$ first, component $B$ second, then sequential combined modularity can be calculated for a two component system as shown in (3):

$$
M_{\text {sys }}\left(\Phi_{A}, \Phi_{B}, \mathbf{p}_{A}, \mathbf{p}_{B}\right)=\int_{\phi_{A}} \int_{\phi_{B}\left(\rho_{A}\right)} d p_{B} d p_{A}
$$

If the order of the update information is not available, modularity calculations for all possible orders (for example, update component $A$ first, component $B$ second and component $B$ first, component $A$ second for a two component scenario) can be added with appropriate weighing coefficients (such as the likelihood of component failure) to calculate the combined modularity of the system.

\section{Optimization Problem to Maximize Combined Compo- nent Swapping Modularity}

In Fig. 1, the block diagram representation for a system consists of a base controller, with transfer function, $\mathbf{C}_{\mathbf{B C}}$, a controlled system, with plant dynamics $\mathbf{P}_{\mathbf{C S}}$, equipped with two smart components, $\mathrm{A}$ and $\mathrm{B}$ is illustrated. We define $\mathbf{p}_{X}$, as a parameter vector representing the component $\mathrm{X}$ plant dynam- 
ics transfer function, $P_{X}$. We also denote nominal settings for these plant parameters as $\mathbf{p}_{X}^{0}$. We can formulate the distribution problem which maximizes swapping modularity of component $\mathrm{A}, M_{A}$, to determine the controller transfer function matrices, $\mathbf{C}_{B C}, \mathbf{C}_{A}, \mathbf{C}_{B}$ as:

$$
\max _{\mathbf{C}_{B C}, \mathbf{C}_{A}, \mathbf{C}_{B}} M_{A}\left(\Phi_{A}, \mathbf{p}_{A}\right)
$$

subject to

$$
C_{\text {dist }}\left(\mathbf{C}_{B C}, \mathbf{C}_{A}, \mathbf{C}_{B}\right)=C_{\text {des }}\left(\mathbf{p}_{C S}^{0}, \mathbf{p}_{A}^{0}, \mathbf{p}_{B}^{0}\right)
$$

$$
\mathbf{g}\left(\mathbf{p}_{C S}, \mathbf{p}_{A}, \mathbf{p}_{B}, \mathbf{C}_{\mathbf{B C}}, \mathbf{C}_{\mathbf{A}}, \mathbf{C}_{\mathbf{B}}\right) \leq \mathbf{0}
$$

where $C_{d e s}$ is the desired centralized controller, determined by any traditional control design method given nominal plant parameters $\mathbf{p}_{C S}^{0}, \mathbf{p}_{A}^{0}, \mathbf{p}_{B}^{0}$. Then, $\mathbf{C}_{\text {dist }}$ is the effective centralized controller calculated from component controllers, $\mathbf{C}_{B C}, \mathbf{C}_{A}, \mathbf{C}_{B}$, and $\mathbf{g}(\mathbf{P}, \mathbf{C})$ in (6) refer to the additional problem specific constraints (e.g., limits on parameters or controller gains).

In order to formulate the combined swapping modularity problem we will use combined modularity, $M_{\text {sys }}$, given in (2) or (3) instead of individual component swapping modularity, $M_{A}$, given in (4).

\section{EXAMPLE: COMBINED COMPONENT SWAPPING MODULARITY OF A VCT ENGINE}

Variable Camshaft Timing (VCT) schemes increase internal residual gas by affecting the intake, combustion and exhaust phases of the engine cycle. Increase in internal residual gas reduces the combustion temperature which decreases nitrogen oxide, $N O_{x}$, formation. The internally recirculated exhaust gas is rich in unburned hydrocarbons, $H C$, which can be burned in the next cycle. Application of VCT schemes, since they require higher manifold pressure, decrease pumping losses which results in improved fuel economy. However, dilution of the incylinder mixture adversely affects the engine torque response. These factors define the trade-off between good emissions and good drivability for VCT engines. Development of a continuous, non-linear, low-frequency, phenomenological and control oriented VCT engine model was given in [5] based on the model structure given in [7] and others.

In [3], the design and distribution of a discrete MIMO controller for a VCT engine is presented. The important steps of the modeling, control design and distribution phases are outlined as an Appendix to this paper for the reader's convenience. The resulting distributed controllers maximize the component swapping modularity of the smart VCT component and smart EGO Sensor (i.e., these components have an on-board computing and

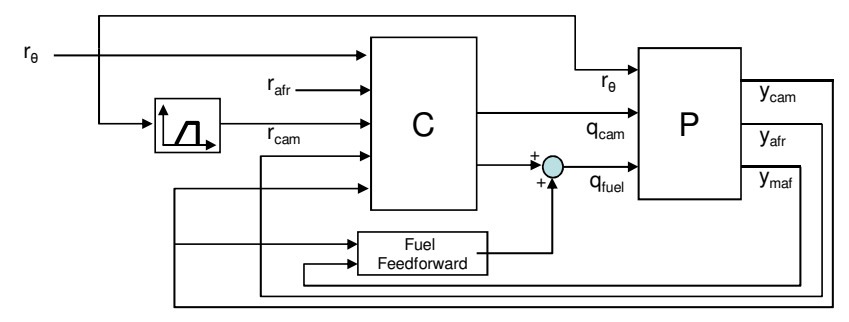

Figure 3. VCT Engine with Discrete MIMO Controller

network connectivity). The block diagram representing the plant and controller relationship for the discrete MIMO controller is given in Fig. 3. As described in [3], centralized controller, $C$, is a transfer function matrix obtained by solving the discrete LQG control design problem. Also, we model the VCT component plant dynamics with parameters $\tau_{v c t, a c t}$ and $\tau_{v c t, s e n}$ (i.e. first order transfer function time constant parameter and a first order Pade approximation parameter respectively). For the EGO sensor plant dynamics, first order dynamics with time constant $\tau_{\text {ego }}$ is used. The objective in solving the controller distribution problem is to find component controllers, $\mathbf{C}_{e c u}, \mathbf{C}_{v c t}, \mathbf{C}_{e g o}$ which improve the component swapping modularity of the system by using bi-directional network communications. Thus, in terms of our earlier notation in Section , $C_{B C}=C_{e c u}$ is the base controller located in the Engine Control Unit (ECU), $C_{A}=C_{v c t}$ is the controller for component A located in the smart VCT component, and $C_{B}=C_{\text {ego }}$ is the controller for component B located in the smart EGO sensor. The block diagram of the proposed distributed system with the proposed communication is given in Fig. 4.

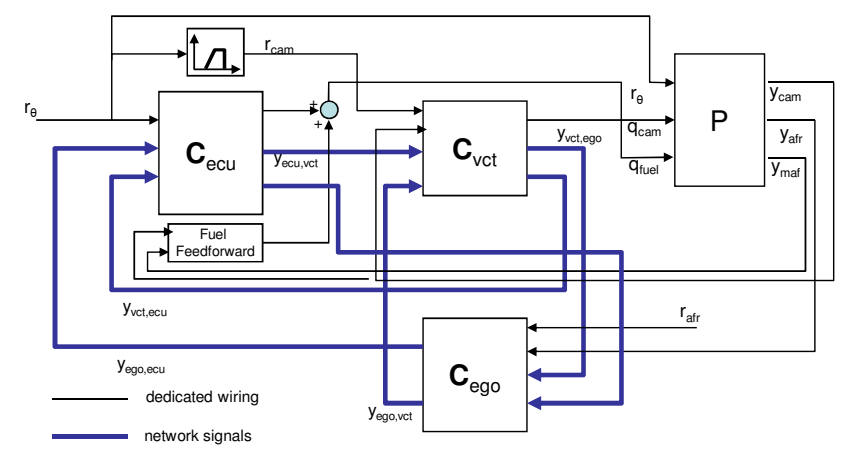

Figure 4. VCT Engine with Distributed Discrete MIMO Controller

By using the same discrete MIMO design methodology described in [3] to calculate $C_{d e s}$ and $C_{d i s t}$, and using one of the combined modularity measures developed in the previous section, it is possible to formulate the design optimization problem that maximizes combined component swapping modularity of the VCT Component and the EGO Sensor. 


\section{Simultaneous Approach}

Calculating the combined swapping modularity of the VCT component and EGO sensor, using the simultaneous approach previously discussed, applies to Cases 2 and 3 in Section where a baseline VCT Engine control design is developed and variants of this design (i.e. with different VCT component or EGO Sensor) will be used in different applications. After the pre-

\begin{tabular}{|l|c||c|c||c|c|}
\hline Element & Solution & Element & Solution & Element & Solution \\
\hline$C_{\text {ecu } 11}$ & $C_{21}$ & $C_{v c t 11}$ & $C_{13}$ & $C_{\text {ego } 11}$ & $C_{22}$ \\
$C_{\text {ecu } 12}$ & 1 & $C_{v c t 12}$ & $C_{15}$ & $C_{\text {ego } 12}$ & $C_{24}$ \\
$C_{\text {ecu } 13}$ & 1 & $C_{v c t 13}$ & $C_{11}-C_{12} / z$ & $C_{\text {ego } 13}$ & 1 \\
$C_{\text {ecu } 21}$ & 1 & $C_{v c t 14}$ & $C_{12}$ & $C_{\text {ego } 14}$ & 0 \\
$C_{\text {ecu } 22}$ & 0 & $C_{v c t 21}$ & $C_{23} z$ & $C_{\text {ego } 21}$ & 1 \\
$C_{\text {ecu } 23}$ & 0 & $C_{v c t 22}$ & $C_{25} z$ & $C_{\text {ego } 22}$ & -1 \\
$C_{\text {ecu } 31}$ & 1 & $C_{v c t 23}$ & 0 & $C_{\text {ego } 23}$ & 0 \\
$C_{\text {ecu } 32}$ & 0 & $C_{v c t 24}$ & 0 & $C_{\text {ego } 24}$ & 1 \\
$C_{\text {ecu33 }}$ & 0 & $C_{v c t 31}$ & 0 & & \\
& & $C_{v c t 32}$ & 1 & & \\
& & $C_{v c t 33}$ & 0 & & \\
& & $C_{v c t 34}$ & 0 & & \\
\hline
\end{tabular}

Table 1. Distribution Solution with Simultaneous Approach

optimization procedure described in [3] is used to obtain a candidate solution for the optimization problem, the distribution solution given in Table 1 is obtained. The solution presented in Table 1 and illustrated in Fig.5 has the optimal combined modularity $M_{s y s}^{*}=613$ based on the measure given in (2) where component modularities $M_{v c t}$ and $M_{\text {ego }}$ have equal weights (i.e. $\left.\rho_{v c t}=\rho_{e g o}=1\right)$. The distributed system shown in Fig. 5 can be reconfigured by only changing the VCT controller, $\mathbf{C}_{v c t}$, for different VCT components with plant dynamic properties $\tau_{v c t, a c t}=[7,67] \mathrm{ms}$ and $\tau_{v c t, s e n}=[10,20] \mathrm{ms}$. Also, the same distributed system can be reconfigured by only changing the EGO controller, $\mathbf{C}_{\text {ego }}$, when different EGO sensors with plant dynamic properties $\tau_{e g o}=[64,77] \mathrm{ms}$ are used. All the resulting systems would have the desired closed loop characteristics with optimal performance.

The distributed controller presented in Table 1 is run in closed loop with the Simulink model of the engine plant model developed. Results of the simulation is then compared to the original centralized MIMO controller and showed no distinguishable difference in response as shown in Fig. 6.

\section{Sequential Approach}

Calculating the combined swapping modularity of the VCT Component and EGO Sensor, using the sequential approach previously discussed, can be used for a case where upgrade and maintenance of an engine system is considered (i.e. Case 1 in Section ). As time progresses, due to failure, or existence of better and cheaper alternatives, components can be changed one at a time and optimal performance is obtained at every step without re-configuring the whole system.

When combined modularity of the VCT Component and EGO Sensor is calculated sequentially two different orders of

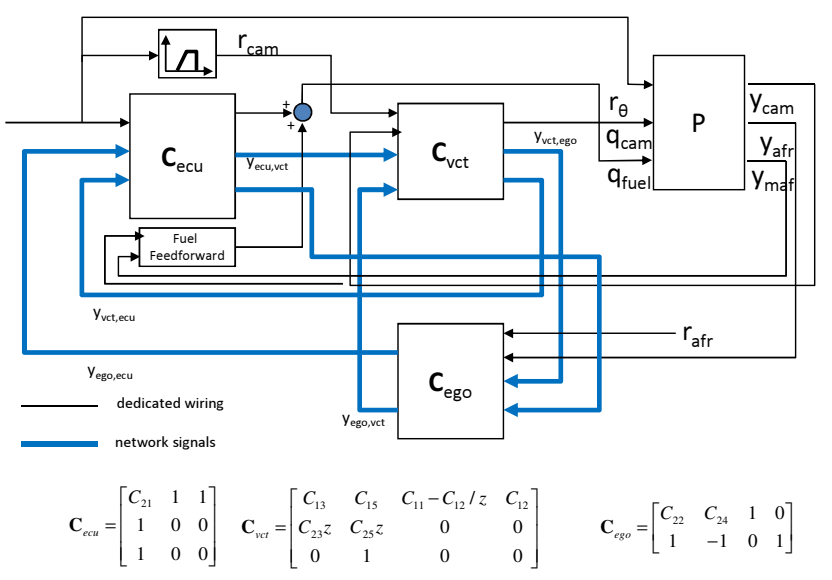

Figure 5. Simultaneous and VCT Component first, EGO Sensor second Sequential Solution.
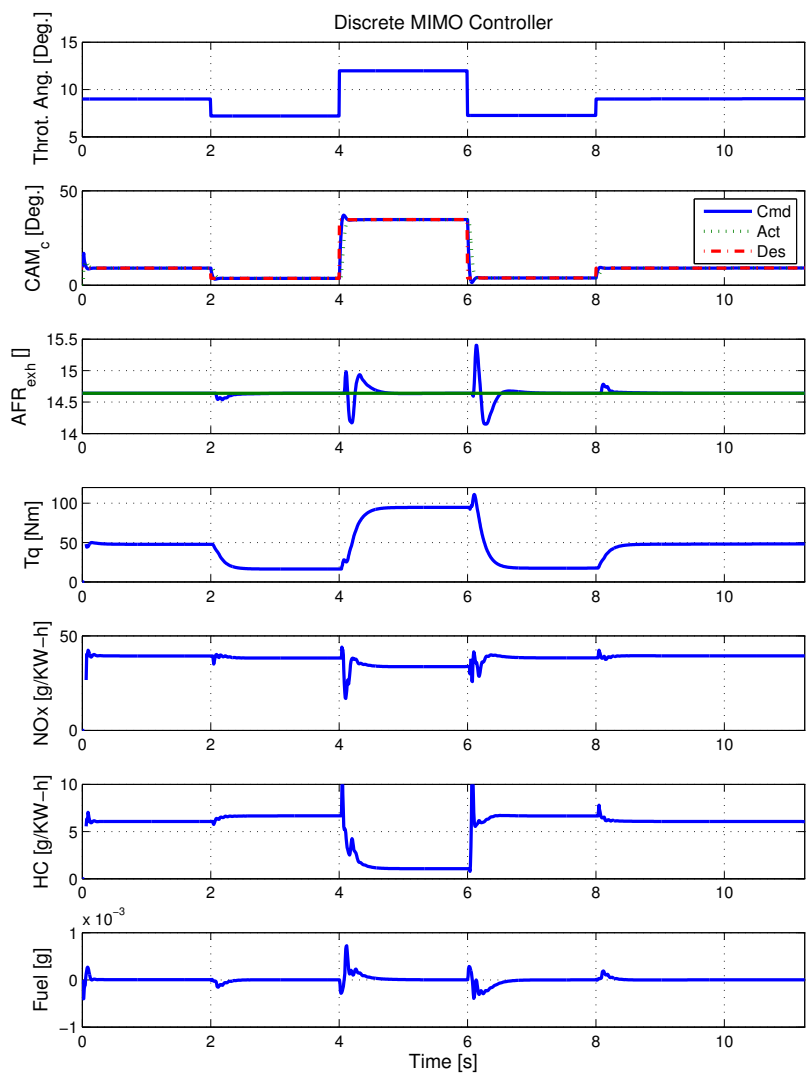

Figure 6. Comparison of Centralized vs. Distributed Controllers

calculation are possible. If the order is upgrade VCT Component first, EGO Sensor second then, after the pre-optimization analysis, the optimal distribution solution obtained is the same as the simultaneous case presented in Table 1. This solution has the combined system modularity of $M_{\text {sys }}^{*}=23.2 \mathrm{e} 3$ based on the sequential measure presented in (3). The modularity value represents an irregularly for shaped volume since it involves ranges 
from three plant parameters. But using a conservative estimate (i.e. giving the maximum dimensions of a rectangular prism that would fit inside this volume), it can be said that VCT components with plant dynamics $\tau_{v c t, a c t}=[7,67] \mathrm{ms}$ and $\tau_{v c t, \text { sen }}=[10,20] \mathrm{ms}$ can be modularly swapped first by only reconfiguring the VCT controller, $C_{v c t}$, then for each configuration, EGO sensors with plant dynamics $\tau_{\text {ego }}=[70,76] \mathrm{ms}$ can be modularly swapped second by only reconfiguring the EGO controller, $C_{\text {ego }}$. All the resulting systems would have the desired closed loop characteristics with optimal performance. When the assumed order is

\begin{tabular}{|l|c||c|c||c|c|}
\hline Element & Solution & Element & Solution & Element & Solution \\
\hline$C_{\text {ecu } 11}$ & $C_{21}$ & $C_{v c t 11}$ & $C_{13}$ & $C_{\text {ego } 11}$ & $C_{22}$ \\
$C_{\text {ecu } 12}$ & 1 & $C_{v c t 12}$ & $C_{15}$ & $C_{\text {ego } 12}$ & $C_{24}$ \\
$C_{\text {ecu } 13}$ & 1 & $C_{v c t 13}$ & 0 & $C_{\text {ego } 13}$ & 0 \\
$C_{\text {ecu } 11}$ & 0 & $C_{v c t 14}$ & 1 & $C_{\text {ego } 14}$ & 0 \\
$C_{\text {ecu } 22}$ & 0 & $C_{v c t 21}$ & 0 & $C_{\text {ego } 21}$ & $C_{12}$ \\
$C_{\text {ecu } 23}$ & 0 & $C_{v c t 22}$ & 0 & $C_{\text {ego } 22}$ & $C_{14}$ \\
$C_{\text {ecu31 }}$ & 1 & $C_{v c t 23}$ & 0 & $C_{\text {ego } 23}$ & 0 \\
$C_{\text {ecu32 }}$ & 0 & $C_{v c t 24}$ & 0 & $C_{\text {ego } 24}$ & $C_{11} z$ \\
$C_{\text {ecu33 }}$ & 0 & $C_{v c t 31}$ & $C_{23} z$ & & \\
& & $C_{v c t 32}$ & $C_{25} z$ & & \\
& & $C_{v c t 33}$ & 0 & & \\
& & $C_{v c t 34}$ & 0 & & \\
\hline
\end{tabular}

Table 2. Distribution Solution with Sequential Approach (EGO first, VCT second)

upgrade EGO Sensor first, VCT Component second then, after the pre-optimization analysis, the optimal distribution solution in Table 2 and Fig. 7 is obtained. The optimal combined system modularity is $M_{s y s}^{*}=8.1 e 3$ based on the sequential measure presented in (3). For this optimal distribution solution, using the same conservative estimate before, it can be said that EGO sensors with plant dynamics $\tau_{\text {ego }}=[64,78] \mathrm{ms}$ can be modularly swapped first by only reconfiguring the EGO controller, $C_{\text {ego }}$, then for each configuration, VCT components with plant dynamics $\tau_{v c t, a c t}=[15,48] \mathrm{ms}$ and $\tau_{v c t, s e n}=[14,20] \mathrm{ms}$ can be modularly swapped second by only reconfiguring the VCT controller, $C_{v c t}$. All the resulting systems would have the desired closed loop characteristics with optimal performance. As in the simultaneous case, the distributed controller presented in Table 2 is run in closed loop with the simulink model of the engine plant model developed. Results of the simulation (see Fig. 6) is then compared to the original centralized MIMO controller and showed no distinguishable difference.

\section{CONCLUSION}

Availability of on-board electronics has increased the use of "smart" components in automatic control systems. The use of bi-directional communications provide additional design freedom which can be used to maximize the swapping modularity of networked smart components. In this paper, application of the design method presented in [1] for combined swapping modularity of system component is discussed. Development of measures

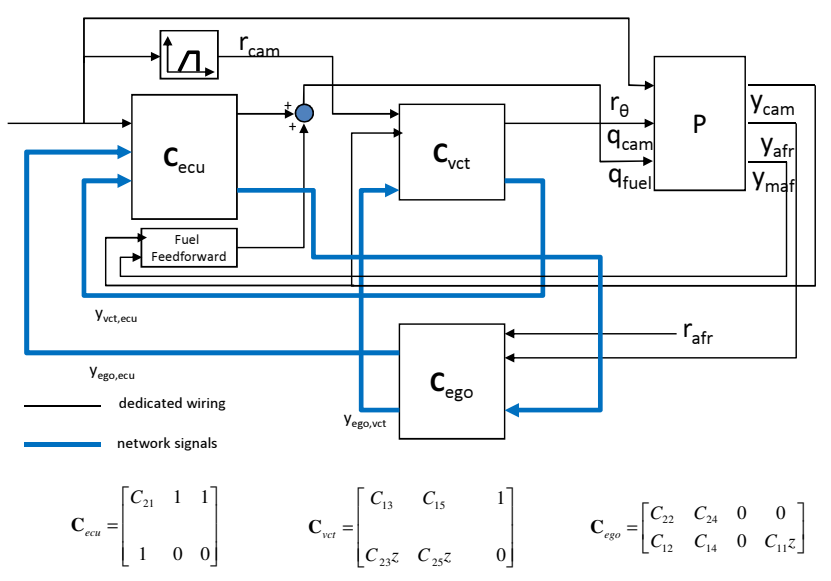

Figure 7. EGO Sensor first, VCT Component second Sequential Solution.

for combined swapping modularity is important to be able to analyze realistic engineering cases. Two approaches (simultaneous and sequential) for combining component swapping modularity of two or more components are presented. These combined modularity approaches are used to design a controller which maximizes the component-swapping modularity of the VCT Component (i.e. actuator and sensor) and EGO Sensor for the example given in [3].

Using the simultaneous combined modularity approach, we have found that the distributed system shown in Fig. 5 can be reconfigured to give optimal controller performance by only changing the VCT controller, $\mathbf{C}_{v c t}$, for different VCT components with time constants in the range $\tau_{v c t, a c t}=[7,67] \mathrm{ms}$ and delay times in the range $\tau_{v c t \text {,sen }}=[10,20] \mathrm{ms}$. The same distributed system can be reconfigured to give optimal controller performance by only changing the EGO controller, $\mathbf{C}_{e g o}$, when different EGO sensors with the delay times in the range $\tau_{\text {ego }}=$ $[64,77] \mathrm{ms}$ are used.

Using the sequential combined modularity approach and assuming the order of upgrades as, VCT component first, EGO sensor second, we have found that with the distributed system shown in Fig. 5, VCT components with the time constants in the range $\tau_{v c t, a c t}=[7,67] \mathrm{ms}$ and delay times in the range $\tau_{v c t, \text { sen }}=$ $[10,20] \mathrm{ms}$ can be modularly swapped first by only reconfiguring the VCT controller, $C_{v c t}$. Then for each of these configurations, EGO sensors with delay times in the range $\tau_{\text {ego }}=[70,76] \mathrm{ms}$ can be modularly swapped second by only reconfiguring the EGO controller, $C_{\text {ego }}$. When we assumed the order of upgrades as, EGO sensor first, VCT component second, EGO sensors with delay times in the range $\tau_{\text {ego }}=[64,78] \mathrm{ms}$ can be modularly swapped first by only reconfiguring the EGO controller, $C_{e g o}$, with the optimal distribution solution given in Fig. 7. Then for each configuration, VCT components with time constants in the range $\tau_{v c t, a c t}=[15,48] \mathrm{ms}$ and $\tau_{v c t, s e n}=[14,20] \mathrm{ms}$ can be modularly swapped second by only reconfiguring the VCT controller, $C_{v c t}$. All the resulting systems would have the desired closed 
loop characteristics with optimal performance.

Our results here show that the combined modularity problem is a more difficult problem then the individual component swapping modularity problem treated previously in [3]. Due to their different units, a fair comparison among the different combined modularity measures (i.e. $M_{s y s}$ simultaneous and sequential) and component swapping modularity, $M_{X}$, is difficult. However, it is seen that the interval of solutions where optimal controllers can be designed is reduced from the results given in [3] (i.e. $\left.\tau_{v c t, a c t}=[7,67] m s, \tau_{v c t, s e n}=[10,20] m s, \tau_{\text {ego }}=[40,100] m s\right) \mathrm{de}-$ spite the increased use of communication paths and higher order transfer function solutions. We also observe that the solutions observed with the sequential approach depend on the sequence of configuration and is not the same as the simultaneous solution.

It is also important to note that distributed controller solutions presented here utilize the bi-directional communications introduced earlier and have improved combined swapping modularity properties than the traditional centralized version of the controller (see Fig. 3) if the VCT component or the EGO Sensor is changed the controller has to be redesigned to achieve desired optimal closed loop performance.

Future research on this topic will include additional applications, as well as improvements to the formulation and solution of the distribution of control problem.

\section{REFERENCES}

[1] M. Cakmakci and A. Ulsoy, "Improving component swapping modularity using bi-directional communication in networked control systems," IEEE Transactions on Mechatronics, vol. 14, no. 3, pp. 307-316, June 2009.

[2] K. Ulrich and K. Tung, "Fundamentals of product modularity," in Issues in Mechanical Design International, A. Sharon, Ed. New York: ASME, 1991, pp. 73-79.

[3] M. Cakmakci and A. G. Ulsoy, "Modular discrete optimal mimo controller for a vct engine," Proceedings of the American Control Conference, June 2009.

[4] A. Stefanopoulou, J. Cook, J. Grizzle, and J. Freudenberg, "Control-oriented model of a dual equal variable cam timing spark ignition engine," ASME Journal of Dynamic Systems, Measurement and Control, vol. 120, pp. 257-266, 1998.

[5] A. Stefanopoulou, "Modeling and control of advanced technology engines," Ph.D. dissertation, University of Michigan, 1996.

[6] A. Stefanopoulou, J. Freudenberg, and J. Grizzle, "Variable camshaft timing engine control," IEEE Transactions on Control Systems Technology, vol. 8, no. 1, pp. 23-34, 2000.

[7] B. Powell and J. Cook, "Nonlinear low frequency phenomenological engine modeling and analysis," Proceedings of the American Control Conference, vol. 24, 1982.

\section{APPENDIX: ENGINE MODELING AND MIMO CONTROL DESIGN AND DISTRIBUTION \\ VCT Engine Model}

The input/output relationship of the plant model developed is given in Fig. 8.

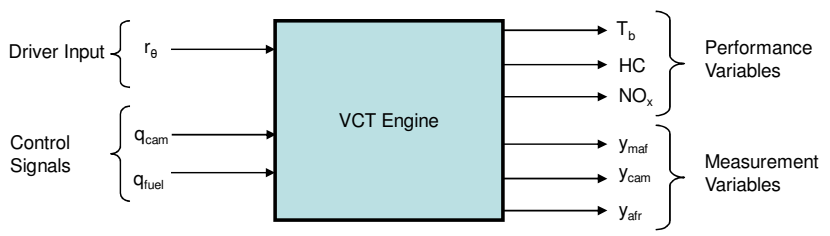

Figure 8. Input Output relationship of the dynamic plant model for control development.

An experimental setup was used to develop relationships for the engine breathing process, torque generation and feedgas $H C$ and $N O_{x}$ emissions were developed. Details of this work will not be discussed here, and the reader is referred to [5] and [4].

In order to model the VCT actuator dynamics, a first order transfer function where $\tau_{v, a}=0.0371$ will be used:

$$
Y_{c, a c t}(s)=\frac{-0.013 s+\tau_{v, a}}{s+\tau_{v, a}} Q_{c}(s)
$$

where $Q_{c}$ is the commanded cam phase angle. For the VCT sensor, a delay of two fundamental sampling periods was assumed, modeled as a first order Pade approximation with parameter $\tau_{v, s}$. For an $n$ cylinder engine at a speed of $N$ rpm the fundamental sampling rate is defined as [5]:

$$
\Delta T=\frac{120}{N n}
$$

The dynamics of the EGO sensor is modeled as first order with a time constant $\tau_{e}=70 \mathrm{~ms}$ :

$$
Y_{a f r}(s)=\frac{1 / \tau_{e}}{s+1 / \tau_{e}} Y_{a f r, e x h}(s)
$$

The Matlab/Simulink plant model for the VCT engine was developed based on the information and regression data given in [5].

\section{Discrete-time MIMO Controller Design}

The dynamic engine model is linearized around the nominal inputs, i.e CAM Angle $=10^{\circ}$, Fuel $=0$ grams and Throttle Angle $=9.33^{\circ}$, and the corresponding steady state internal states using Matlab/Simulink. 
The linearized model is then discretized with a sampling pe$\operatorname{riod} \Delta T$ to obtain

$$
\begin{array}{r}
\mathbf{x}(k+1)=\mathbf{A}_{\mathbf{d}} \mathbf{x}(k)+\mathbf{B}_{\mathbf{d}} \mathbf{u}(k)+\mathbf{B}_{\mathbf{r} \mathbf{1}, \mathbf{d}} r_{\theta}(k) \\
\mathbf{y}(k)=\mathbf{C}_{\mathbf{d}} \mathbf{x}(k)+\mathbf{D}_{\mathbf{d}} \mathbf{u}(k)+\mathbf{B}_{\mathbf{r} \mathbf{1}, \mathbf{d}} r_{\theta}(k)
\end{array}
$$

where

$$
\begin{aligned}
& \mathbf{A}_{d}=\left[\begin{array}{ll}
\mathbf{A}_{d 1} & \mathbf{A}_{d 2}
\end{array}\right] \\
& \mathbf{A}_{d 1}=\left[\begin{array}{ccccc}
0.8984 & -0.01638 & 0.02042 & 0.0025 & 0.0003 \\
0 & 0.8169 & 0 & 0 & 0 \\
0 & 0.1153 & 0.3679 & 0 & 0 \\
0 & -0.00107 & 0.0307 & 0.9435 & 0.022 \\
0 & 0 & 0 & 0 & 0.7575 \\
0 & 0.0045 & 0 & 0 & 0 \\
0 & 1 e-6 & -7 e-5 & 0.0002 & 1 e-5 \\
0 & -0.0238 & -0.0032 & 0.0456 & 0.0054 \\
0 & -7 e-5 & -0.0003 & 0.0005 & 5 e-5
\end{array}\right] \\
& \mathbf{A}_{d 2}=\left[\begin{array}{cccc}
0.1543 & -656.314 & -0.8944 & 10.1589 \\
0 & 0 & 0 & 0 \\
0 & 0 & 0 & 0 \\
0 & 0 & 0 & 0 \\
0 & 0 & 0 & 0 \\
0.944 & 0 & 0 & 0 \\
0 & 0.3679 & 0 & 0 \\
0.2551 & -1240 & 0.466 & -14.0665 \\
0.0011 & -6.3646 & 0.0053 & 0.9407
\end{array}\right] \\
& {\left[\mathbf{B}_{r 1, d}, \mathbf{B}_{d}\right]=\left[\begin{array}{ccc}
0.00038175 & -5.354 e-5 & 0.14931 \\
0 & 0.0067904 & 0 \\
0 & 0.00046453 & 0 \\
0.00024734 & -7.5544 e-6 & 0 \\
0.018606 & 0 & 0 \\
0.0024429 & 1.5316 e-5 & 0 \\
2.2866 e-7 & 2.2808 e-8 & 0.0047409 \\
0.00052672 & -6.6406 e-5 & 1.4671 \\
1.5265 e-6 & -1.3251 e-7 & 0.014705
\end{array}\right]} \\
& \mathbf{C}_{d}=\left[\begin{array}{ccccccccc}
14.2857 & 0 & 0 & 0 & 0 & 0 & 0 & 0 & 0 \\
0 & -27.310 & 266.667 & 0 & 0 & 0 & 0 & 0 & 0
\end{array}\right] \\
& \mathbf{D}_{d}=\left[\begin{array}{ccc}
0 & 0 & 0 \\
0 & 0.013 & 0
\end{array}\right]
\end{aligned}
$$

The state vector is augmented with the integral of the output tracking errors:

$$
\begin{aligned}
\hat{\mathbf{x}}(k+1)= & {\left[\begin{array}{cc}
\mathbf{A}_{\mathbf{d}} & \mathbf{0} \\
\Delta T \mathbf{C}_{\mathbf{d}} & \mathbf{I}
\end{array}\right]\left[\begin{array}{c}
\mathbf{x}(k) \\
\mathbf{x}_{\mathbf{I}}(k)
\end{array}\right]+\left[\begin{array}{c}
\mathbf{B}_{\mathbf{d}} \\
-\Delta T \mathbf{C}_{\mathbf{d}}
\end{array}\right] \mathbf{u}(k) } \\
& +\left[\begin{array}{cc}
\mathbf{B}_{\mathbf{r} \mathbf{1}, \mathbf{d}} & \mathbf{0} \\
\mathbf{0} & -T_{s} \mathbf{I}
\end{array}\right]\left[\begin{array}{c}
r_{\theta}(k) \\
r_{c a m}(k) \\
r_{a f r}(k)
\end{array}\right]
\end{aligned}
$$

By using the discrete-time linear system above an LQR controller with the state feedback gains, $K_{d}$ was obtained:

$$
\mathbf{K}_{d}^{T}=\left[\begin{array}{cc}
-0.00029813 & -0.013001 \\
22.2911 & 0.0006195 \\
126.643 & -0.0087846 \\
2.84 e-5 & 0.025793 \\
-2.79 e-6 & 0.0030435 \\
-0.00010603 & -0.038505 \\
1.6371 & 65.1827 \\
-0.00029203 & -0.0079145 \\
-0.065519 & -2.647 \\
0.0001704 & 0.0085496 \\
-40.1194 & 0.00095088
\end{array}\right]
$$

A Kalman Filter is designed to estimate the remaining states with the gains:

$$
\mathbf{L}_{d}=\left[\begin{array}{cc}
0.095152 & 8.486 e-5 \\
-9.9756 e-11 & 0.00020019 \\
-1.8683 e-11 & 0.0014151 \\
-2.6077 e-8 & 0.00012182 \\
-1.0599 e-10 & -4.7379 e-15 \\
2.8741 e-8 & 1.495 e-005 \\
-9.9113 e-7 & -2.9073 e-7 \\
-0.015256 & -6.9183 e-6 \\
0.00014419 & -1.3294 e-6
\end{array}\right]
$$

\section{Distribution Problem}

With the controller distribution problem our aim is to find component controllers, $\mathbf{C}_{e c u}, \mathbf{C}_{v c t}, \mathbf{C}_{e g o}$ which improve the component swapping modularity of the system by using bidirectional network communications. The block diagram of the proposed distributed system with the proposed communication is given in Fig. 4. Given nominal settings for the plant parameters (denoted as $\mathbf{p}_{c s}^{0}, \mathbf{p}_{v c t}^{0}, \mathbf{p}_{\text {ego }}^{0}$ for the controlled system i.e., rest of the engine, VCT component and EGO sensor, respectively), we can formulate the distribution problem which maximizes VCT component swapping modularity, $M_{v c t}$ while the distribution constraint, desired overall controller must be equal to the overall effect of the distributed controller (i.e $C_{d e s}=C_{d i s}$ ) holds. 